\title{
Evaluating the Effect of Interface Shape on the fracture properties of Cellulose Nanocrystal (CNC) Nano-Films
}

\author{
Jim Huang ${ }^{1}$ and Mehrdad Bor ${ }^{1}$ \\ ${ }^{1}$ School of Mechanical and Design Engineering \\ Portsmouth, United Kingdom
}

\begin{abstract}
Crystalline nanocellulose $(\mathrm{CNC})$ is a new promising green material that gained significant attention in the past decade. This study evaluates the effects of pattern interfaces on the fracture toughness in naturally joined double cantilever beam of crystalline nanocellulose (CNC) nanofilms. Three different patterns, e.i., triangular, trapezoidal and circular with different geometric properties were considered and compared with flat (no pattern) interface. We use a multiscale framework by using adhesion and elastic properties are nanoscale from molecular dynamics and upscale the values to the properties of cohesive element in finite element framework. The result shows that pattern interfaces significantly improve the toughness of CNC nanofilms. In particular, trapezoidal interface increase the fracture toughness by $140 \%$.
\end{abstract}

Keywords: Double cantilever beam, Pattern interfaces; Cohesive elements, Multiscale modeling.

\section{INTRODUCTION}

Cellulose is the most abundant polymer on the planet [1], [2]. In the past decade new particles of cellulose called crystalline nanocellulose $(\mathrm{CNC})$ with outstanding mechanical properties received tremendous attention for packaging and reinforcement agent [1]-[3]. As the interfaces between materials often control the macroscopic properties of the composites [4], [5], many studies have been dedicated to characterizing interface properties and find methods to improve the interface properties [6]-[8]. Inspired by natural biological materials and patterned interfaces found in biomineralized interfaces, many researchers have studied the effect of pattern interfaces on the fracture properties of materials and showed that the pattern interface can significantly improve fracture properties [9]-[11]. For example, recently, Hosseini et al (2019), studied the effect of the shape of interface behind the crack tip for triangular, arbitrary, and sinusoidal patterned interfaces and show that for critical values of interfaces the crack could propagate in the bulk material as opposed to the interface [11]. This is particularly important for CNC nanofilms, as aligned CNCs are extremely brittle [12]. In the present work we assume that the crack can only propagate at the interface and leave the bulk material intact. However, we assume length scale to be more critical aspect for fracture properties of $\mathrm{CNC}$ nanofilms, as suggested by Hosseini et al (2019), and as such we use a multiscale framework to consider the effect of nanoscale adhesion and macroscopic properties. Upscaling the mechanical and interfacial properties from Nano to macroscale has been done by many researchers before for CNC [13] and carbon nanotube [14]. For example, Shishehbor et al. (2018), used a novel atomistic to continuum framework for evaluating the role of bonded and non-bonded interactions on the elastic properties of CNCs and showed covalent bond in the main contributor interaction in both bending and tensile loading [13]. We have to mention that, in addition to multiscale framework, coarse grained (CG) molecular dynamics have been used extensively to bridge the scale from nano to macro [15]-[18]. For example, Ramezani and Golchinfar, (2019) used CG modeling for evaluating the fracture properties of CNC bundles and showed that by increasing the bundle size and twist angle strength and toughness decreases [16]. Shishehbor and Zavattieri (2019), studies the effect of interface properties of CNCs in bio-inspired architecture of CNC particles by a new promising CG model and showed that the natural twist of CNCs optimizes the fracture properties of neat CNC materials [17].

From the various test specimens that have been used to measure the adhesive fracture energy, the double-cantilever beam (DCB) specimen, has been one of the most popular. In this work, three different interface shapes were analyzed (triangular, trapezoidal and circular) and compared for mode I fracture in a simple DCB (Double Cantilever Beam) test specimen. For each pattern, a few geometric variation is also performed to provide more insight to the problem. We use finite element (FEM) framework and cohesive element method to analyze DCB specimens at microscale, while the properties if the bulk and interface is provided by atomistic molecular modeling from literature. 


\section{Materials AND Method}

\section{A. Crystalline nanocellulose (CNC)}

CNCs are crystalline structures of cellulose formed when many cellulose chains assembled through hydrogen bonding and formed a crystalline structure with unit cell values of $\mathrm{a}=7.784 \AA$, $\mathrm{b}=8.201 \AA, \mathrm{c}=10.380 \AA, \alpha=90^{\circ}, \beta=90^{\circ}$ and $\gamma=96.55^{\circ}$ as shown in Fig. $2 b$ [1]. The structure of CNC is square shape with 36 chains and dimensions of $3.5 \times 3.5 \mathrm{~nm}^{2}$ and length of $50-500 \mathrm{~nm}$. The reported elasticity modulus of $\mathrm{CNC}$ in the lateral direction from experiments is 10-30 GPa [19], while it is in 5-25 GPa range from numerical methods [1] . In addition, the adhesion properties of $\mathrm{CNC}$ obtained from simulation is reported to be around 100 $(\mathrm{mJ} / \mathrm{m} 2)$ with strength of $0.4 \mathrm{GPa}$ [17]. In the following sections, we use these nanoscale properties to feed DCB properties in the FEM framework.

\section{B. Multiscale famework}

A DCB specimen used as the main geometry in this work is shown in Fig. 1a. Three pattern interfaces are analysize and compared with no-pattern interface results. The distance between the two beams was set to $100 \mathrm{~nm}(0.1 \mu \mathrm{m})$ as previous studies showed that having such small thickness would be equal to having zero thickness effect [11]. Fig.1 demonstrates the DCB specimen of CNC nanofilm with no-pattern interface. The same geometry values for all the pattern interfaces with addition of pattern interfaces in shape of triangular, trapezoidal and circular (the no-pattern interface is shown with blue rectangular shape in Fig. 1a).

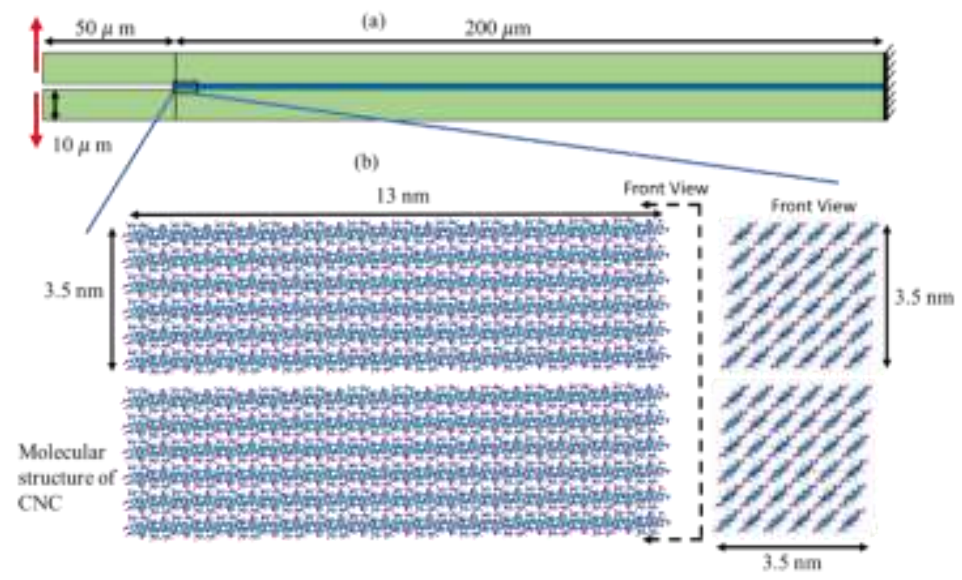

Figure 1: (a) DCB specimen of CNC nanofilm with boundary condition. (b) Molecular structure of CNC from the side and front view.

\section{Pattern interfaces}

The second specimen consists in the original DCB beam with a change in the geometry of the adhere surfaces. A triangular pattern was applied to the section of the beams but keeping the initial crack as a straight line. The four variations of this shape that were tested are presented in Error! Reference source not found.. Each of the shapes is referred using a unique identification code that is present in the top edge of the figure. The third model consists in a trapezoidal shape. This subtype was introduced to soften the stress concentration effects that sharp tips present in triangles will generate over the beam. As it was done before, the predefined crack remains as a straight line. The four variations of this shape that were tested are presented in Error! Reference source not found. Each of the shapes is referred using a unique identification code that is present at the top edge of the figure. The final model uses a circular pattern. This shape reduces the stress concentration generated by the previous geometries but still producing a significant deviation in the crack path. The two variations of this shape that were tested are presented in Error! Reference source not found. Each of the shapes is referred using a unique identification code that is present at the top edge of the figure. It is important to notice that the geometry changes introduced to the section shape of the beams will generate changes in the crosssection properties (moment of inertia and area).

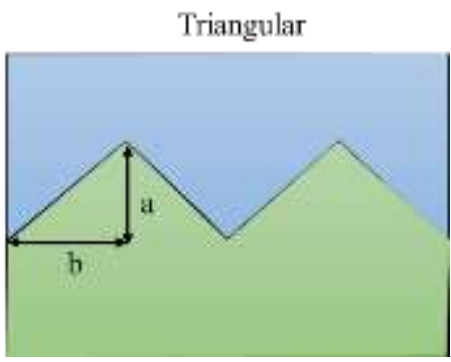

Trapezoidal

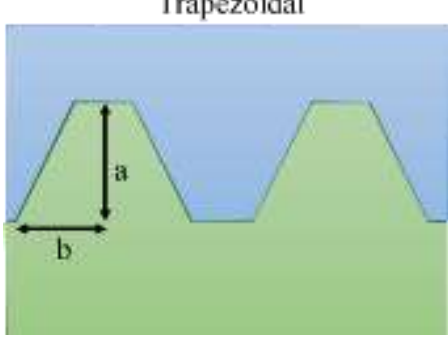


(a)

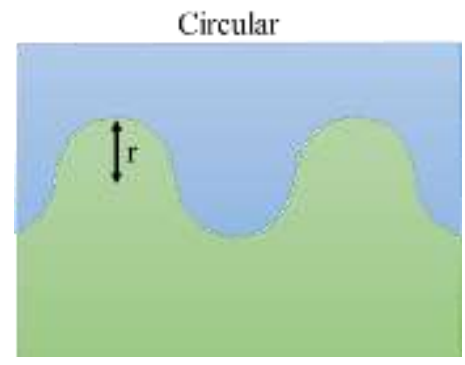

(c)

(b) ameters studied in this work. (a) Triangular, (b) Trapezoidal and

(c) Circular.

\section{Cohesive zone model}

Cohesive Zone model is one the most powerful models in the fracture mechanics and finite element framework for incorporating fracture process and modeling crack propagation. For example, Esmaeeli et al, used a multiscale cohesive zone model for predicting fracture of cementitious composites at early age by inserting cohesive elements between cement and aggregates and showed that crack can propagate both in cement and aggregate depending on the cement age [20]. We use cohesive elements to define the adhesion between two beams and he parameters that define the used law were selected based on the fracture toughness of CNC. Fig. 3a shows the bi-linear law that is commonly used for brittle solids and the area below the curve shows the fracture toughness of the material. Fig. $3 b$ demonstrates the insertion of cohesive element between two solid triangular element. The properties of the bulk and cohesive element are presented in Table 1.

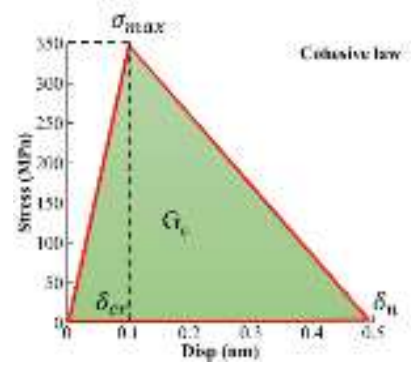

(a)

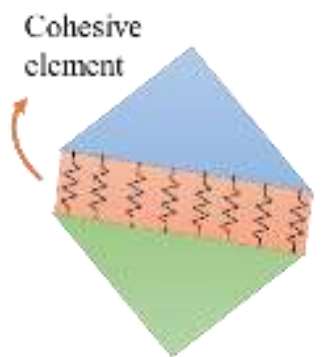

(b)

Figure 3: (a) Bi-linear traction-separation law used in the cohesive zone model (b) The cohesive element (Orange rectangular element) is embedded between traditional elements.

Table1. Mechanical and interfacial propretores of CNC film used for bulk and cohesive respectively.

\begin{tabular}{c|c|c|c|c|c|c|c|}
\hline Properties: & E $(\mathrm{GPa})$ & $\boldsymbol{v}$ & $\gamma(\mathrm{mJ} / \mathrm{m} 2)$ & $\sigma(\mathrm{MPa})$ & $\delta_{c r}(\mathrm{~nm})$ & $\delta_{n}(\mathrm{~nm})$ \\
\hline Values & 20 & 0.3 & 100 & 350 & 0.1 & 0.5 \\
\hline
\end{tabular}

\section{E. FEM package and protocol}

2D plane strain model with ABAQUS standard library elements for bulk and cohesive elements were used in this work. Elements in the solid material were represented by 3-node linear triangle continuum elements (CPEG6) and the interface was represented by a 4-node interface element (COH2D4). Mesh sensitivity analysis has been done for different mesh refinement to find the accurate and computationally affordable mesh. We found meshes with 30,000-40,000 number of element to be accurate and affordable for our analysis (final mesh for all the interfaces are shown in Fig. 4). For loading condition, a total displacement of $5 \mu \mathrm{m}$ ( $2.5 \mu \mathrm{m}$ for top and bottom surface) was applied incrementally. All the simulations are performed using ABAQUS non-linear static analysis with 20,000 total increments and maximum increment of 0.005 were used. 
(a)
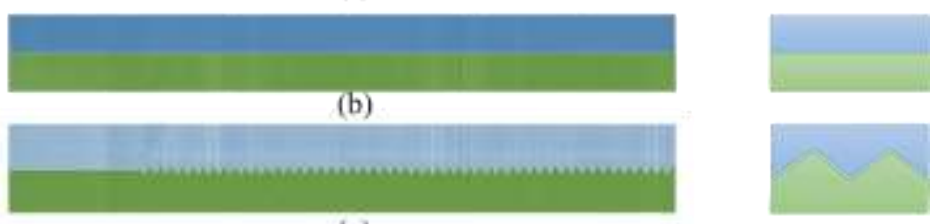

(c)
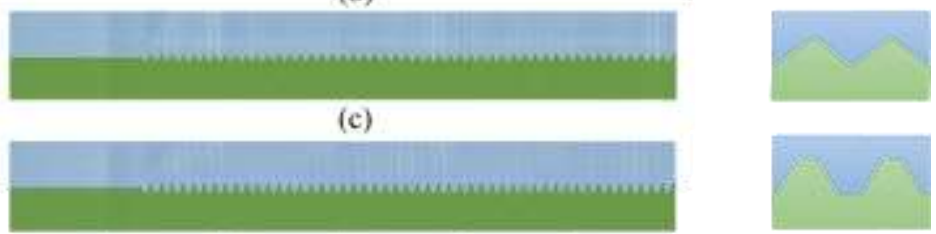

(d)
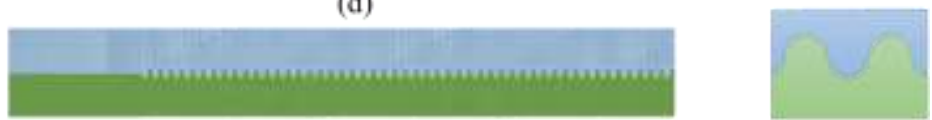

Figure 4: Selected fine mesh for this study. (a) No pattern. (b) Triangular pattern. (c) Trapezoidal pattern. (d) Circular pattern.

\section{RESULTS}

This section represents the results from our analysis. The section has been divided into sub sections for each pattern interface. In each section, the force-half displacement (upper beam), and dissipated energy-crack length plots are presented. Also, the value of toughness is compared with the fracture toughness of the interface $\left(\mathrm{G}_{\mathrm{c}}\right.$, the area below cohesive law). First, the no pattern interface is used as the benchmark and validation point, then the pattern interfaces are represented and finally all the pattern and no pattern interfaces are compared.

\section{A. Validation of DCB test for no pattern interface}

No pattern interface geometry is the best candidate to validate our model setup and assumptions. It is expected that the value of fracture toughness for no patter interface to be approximately equal to the $\mathrm{G}_{\mathrm{c}}$. Fig. 5a and Fig. 5b show the force (P)-half displacement $(\delta)$ and energy dissipation-crack length respectively for the no pattern interface. The obtained value for fracture toughness from the slope of the dissipated energy-crack length curve is compared with $\mathrm{G}_{\mathrm{c}}$ in table 2 . The result shows that the model can accurately predict $\mathrm{G}_{\mathrm{c}}$ with less than $1 \%$ error.

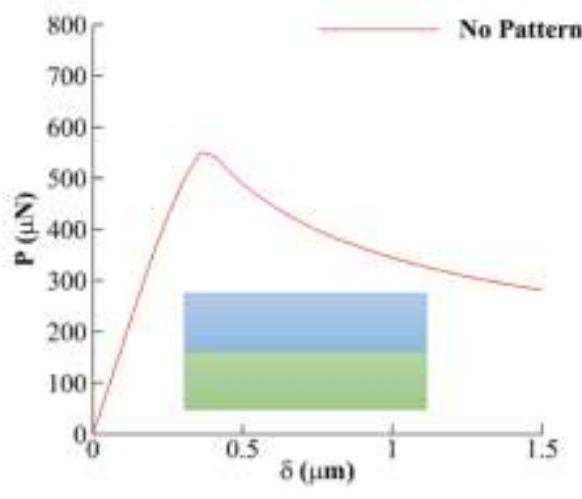

(a)

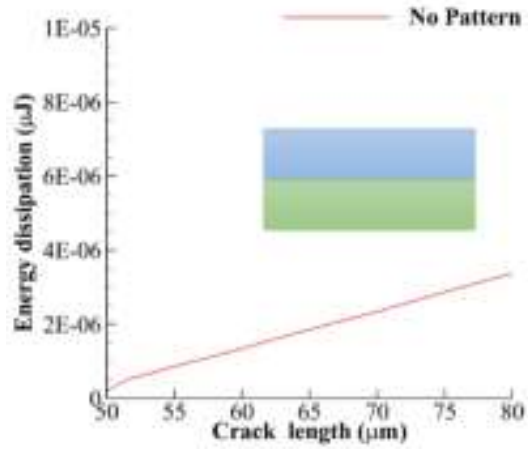

(b)

Figure 5: (a) force (P)-half displacement for no pattern interface (b) Dissipated energy through cohesive element as the crack grows.

Table 2: Fracture tougness from DCB test $(\gamma)$ are comparad fracture toughness of material Gc.

Case $\quad \operatorname{Gc}(\mathrm{mJ} / \mathrm{m} 2) \quad \gamma(\mathrm{mJ} / \mathrm{m} 2) \quad$ Error $(\%)$




\begin{tabular}{l|l|r|r|}
100 & 99.0 & 1.0
\end{tabular}

\section{B. Pattern interfaces}

\section{III.B.1 Triangular pattern interface}

For triangular pattern interfaces, four specimens with changing the geometric parameters, e.i., $a$ and $b$ which represent the height and side length of the triangle, are shown in Fig. 6. Fig. 6a and Fig. 6b show the force (P)-half displacement $(\delta)$ and energy dissipation-crack length respectively for the triangular pattern interfaces. The obtained value for fracture toughness from the slope of the dissipated energy-crack length curve is compared with $\mathrm{G}_{\mathrm{c}}$ in table 3 for different $a$ and $b$ values. The result shows that triangular interfaces significantly improve the fracture toughness of the material and for $a=3$ and $b=2,120 \%$ improvement was observed (Table. 3).The results also indicate that by increasing the height $(a)$ rather than side $(b)$, higher fracture toughness is achievable. It is worth mentioning that both beams are elastic and therefore we ignored the effect of higher stress concentration for triangular interface due to sharp corners.

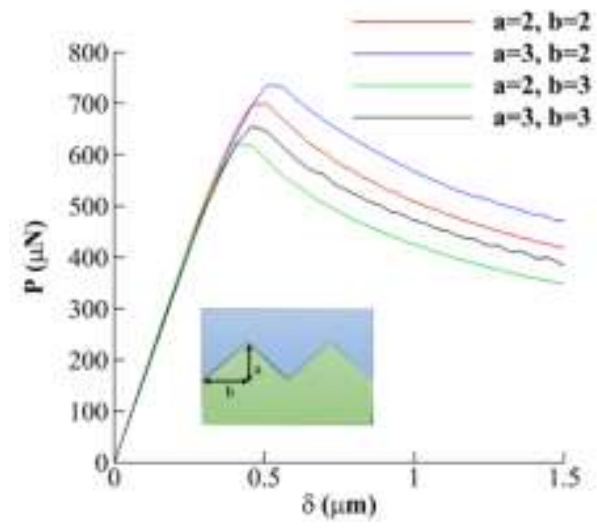

(a)

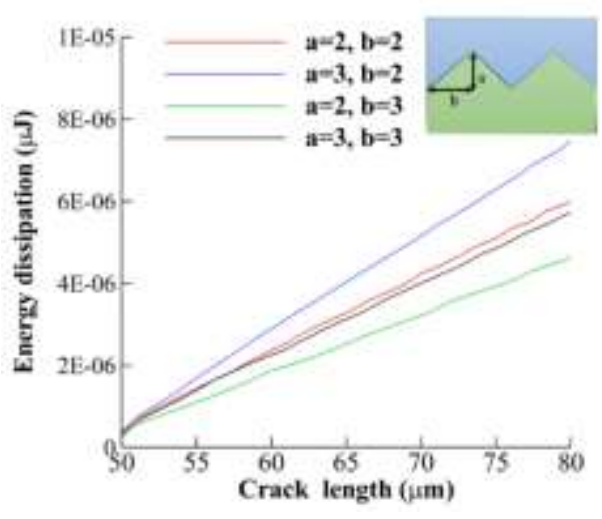

(b)

Figure 6: (a) force (P)-half displacement for triangular pattern interface (b) Dissipated energy through cohesive element as the crack grows for triangular pattern interface.

Table 3: Fracture tougness from DCB test $(\gamma)$ are comparad fracture toughness of material Gc.

\begin{tabular}{|c|c|c|c|c|c|}
\hline Case & $\mathbf{a}(\mathbf{n m})$ & $\mathbf{b}(\mathbf{n m})$ & $\mathrm{Gc}(\mathbf{m J} / \mathbf{m} 2)$ & $\gamma(\mathbf{m J} / \mathbf{m} 2)$ & Error $(\%)$ \\
\hline 1 & 2 & 2 & 100 & 182 & 82.0 \\
\hline 2 & 3 & 2 & 100 & 220 & 120.0 \\
\hline 3 & 2 & 3 & 100 & 135 & 35 \\
\hline 4 & 3 & 3 & 100 & 168 & 68 \\
\hline
\end{tabular}

\section{Trapezoidal pattern interface}

For trapezoidal pattern interfaces, four specimens with changing the geometric parameters, e.i., $a$ and $b$ which represent the height and side length of the trapezoid, are shown in Fig. 7. Fig. 7a and Fig. 7b show the force (P)-half displacement $(\delta)$ and energy dissipation-crack length respectively for the triangular pattern interfaces. The obtained value for fracture toughness from the slope of the dissipated energy-crack length curve is compared with $\mathrm{G}_{\mathrm{c}}$ in table 3 for different $a$ and $b$ values. The result shows that trapezoidal interfaces significantly improve the fracture toughness of the material and for $a=3$ and $b=2,140 \%$ improvement was observed (Table. 4). The results also indicate that by increasing the height $(a)$ rather than side $(b)$, higher fracture toughness is achievable. It is worth mentioning that both beams are elastic and therefore we ignored the effect of higher stress concentration for triangular interface due to sharp corners. This trend of results is very similar to those observed in triangular pattern interface. 


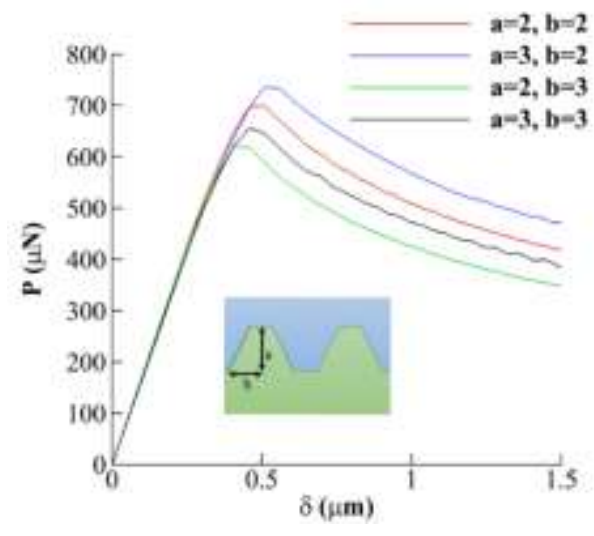

(a)

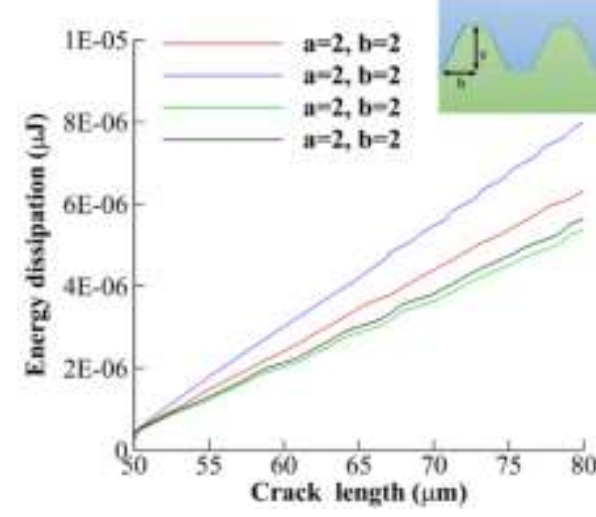

(b)

Figure 7: (a) force (P)-half displacement for trapezoidal pattern interface (b) Dissipated energy through cohesive element as the crack grows for trapezoidal pattern interface.

Table 4: Fracture tougness from DCB test $(\gamma)$ are comparad fracture toughness of material Gc.

\begin{tabular}{|c|c|c|c|c|c|}
\hline Case & $\mathbf{a}(\mathbf{n m})$ & $\mathbf{b}(\mathbf{n m})$ & $\mathrm{Gc}(\mathbf{m} \mathbf{J} / \mathbf{m} \mathbf{2})$ & $\gamma(\mathbf{m} \mathbf{J} / \mathbf{m} \mathbf{2})$ & Error $(\%)$ \\
\hline 1 & 2 & 2 & 100 & 195 & 95 \\
\hline 2 & 3 & 2 & 100 & 240 & 140 \\
\hline 3 & 2 & 3 & 100 & 150 & 50 \\
\hline 4 & 3 & 3 & 100 & 160 & 60 \\
\hline
\end{tabular}

\section{Circular pattern interface}

For Circular pattern interfaces, two specimens with changing the geometric parameters $r$ (which represent the radius of circle) are shown in Fig. 6. Fig. 6a and Fig. 6b show the force $(\mathrm{P})$-half displacement $(\delta)$ and energy dissipation-crack length respectively for the circular pattern interfaces. The obtained value for fracture toughness from the slope of the dissipated energy-crack length curve is compared with $\mathrm{G}_{\mathrm{c}}$ in table 5 for different $r$ values. The result shows that circular interfaces can improve the fracture toughness by $80 \%$ for $r=3$ (Table. 3 ). The circular shape shows a smaller increase in the fracture energy compared to the other geometries, but due to less sharp corners, less stress intensity factor is observed.

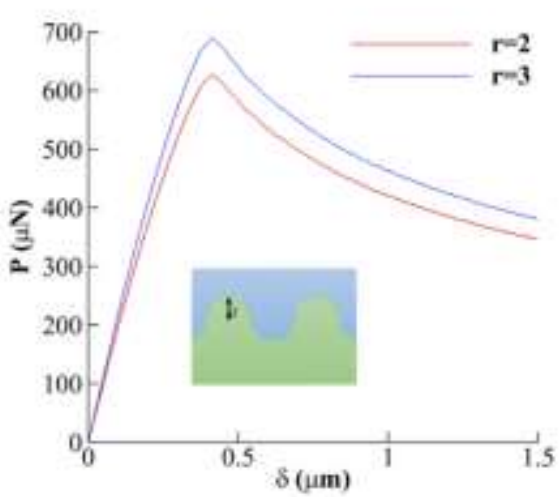

(a)

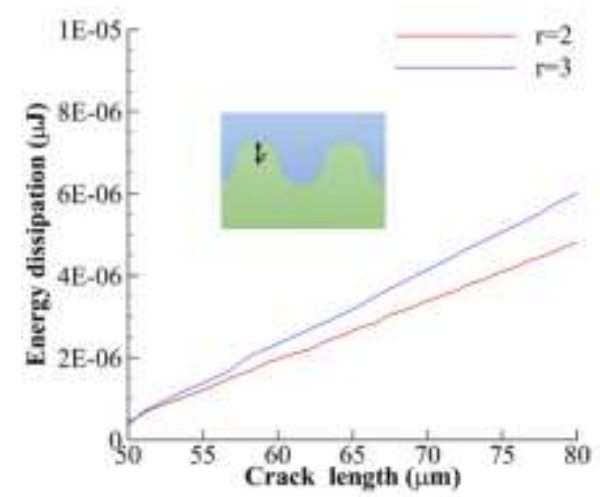

(b)

Figure 8: (a) force (P)-half displacement for circular pattern interface (b) Dissipated energy through cohesive element as the crack grows for circular pattern interface.

Table 5: Fracture tougness from DCB test $(\gamma)$ are comparad fracture toughness of material Gc. 
International Journal of Advances in Scientific Research and Engineering (ijasre), Vol 5 (8), August-2019

\begin{tabular}{|c|c|c|c|c|}
\hline Case & $\mathrm{r}(\boldsymbol{\mu} \mathrm{m})$ & $\mathrm{Gc}(\mathrm{mJ} / \mathrm{m} 2)$ & $\gamma(\mathrm{mJ} / \mathrm{m} 2)$ & Error (\%) \\
\hline 1 & 2 & 100 & 145 & 45 \\
\hline 2 & 3 & 100 & 180 & 80 \\
\hline
\end{tabular}

\section{E. Comparing different patterns}

Fig. 8a and Fig. 8b compare the force (P)-half displacement $(\delta)$ and energy dissipation-crack length respectively for the all interfaces of this study. The obtained value for fracture toughness from the slope of the dissipated energy-crack length curve is compared with $\mathrm{G}_{\mathrm{c}}$ in table 6 for highest fracture energy of different pattern interfaces ( $a=3, b=2$ and $r=3$ ). The result indicate that trapezoidal interface has the highest fracture toughness, then triangular, then circular and finally the no pattern interface. However, for all pattern interfaces, the result showed significant improvement. The force-displacement shows that triangular and trapezoidal have very similar behavior with very slight improvement in trapezoidal.

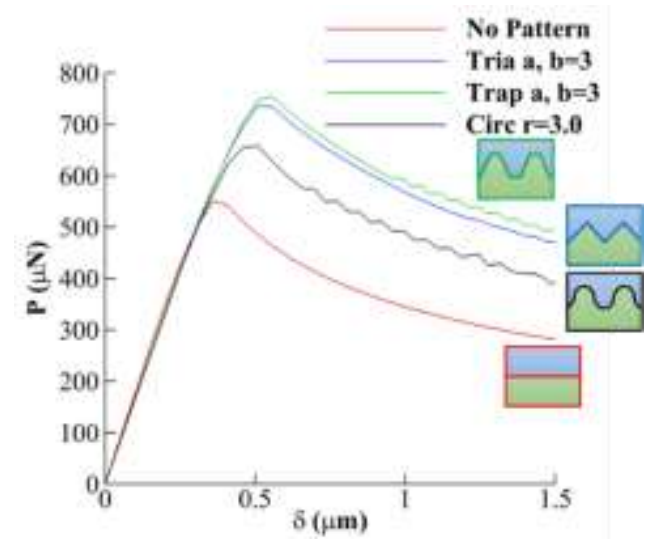

(a)

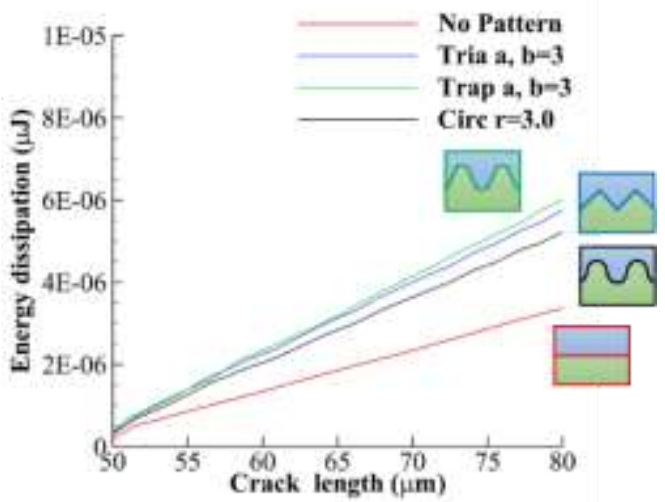

(b)

Figure 8: (a) force (P)-half displacement for all interfaces (b) Dissipated energy through cohesive element as the crack grows for all interfaces.

Table 6: Fracture tougness from DCB test $(\gamma)$ are comparad fracture toughness of material Gc.

\begin{tabular}{|l|l|l|l|}
\hline Case & Gc $(\mathbf{m J} / \mathbf{m} 2)$ & $\gamma(\mathbf{m J} / \mathbf{m} 2)$ & Error $(\%)$ \\
\hline No pattern & 100 & 99 & 1 \\
\hline Triangular & 100 & 220 & 120.0 \\
\hline Trapezoidal & 100 & 240 & 140.0 \\
\hline Circular & 100 & 180 & 80
\end{tabular}

\section{CONCLUSION AND DISCUSSION}

In this work the effects of three pattern interfaces with different geometric parameters on the fracture toughness for a double cantilever beam was presented. Results show that all the pattern interfaces could improve the fracture toughness, while the maximum improvement was $140 \%$ for trapezoidal interface with $a=3$ and $b=2$. Therefore, for brittle polymers such as highly aligned CNCs, using pattern interfaces could help to prevent early crack propagation and failure. There were many assumption and limitation for this study that could be extended in the future. For example, we assume the properties of the beams to be elastic and no fracture was assumed in the bulk material. As a result the stress concentration produced by the edges of the pattern did not have any effect on the results. Considering the crack to be able to propagate in the bulk will provide more realistic study. This study only focuses on the mode I fracture properties, while considering mode II could be as important as mode I and could be an interesting to study.

\section{REFERENCES}

[1] R. J. Moon, A. Martini, J. Nairn, J. Simonsen, and J. Youngblood, "Cellulose nanomaterials review: Structure, properties 
and nanocomposites," Chem. Soc. Rev., vol. 40, no. 7, pp. 3941-3994, Jul. 2011.

[2] R. J. Moon, G. T. Schueneman, and J. Simonsen, "Overview of Cellulose Nanomaterials, Their Capabilities and Applications," Jom, vol. 68, no. 9. pp. 2383-2394, 2016.

[3] D. C. Potulski, G. I. B. De Muniz, U. Klock, and A. S. De Andrade, "Influência da incorporação de celulose microfibrilada nas propriedades de resistência mecânicas do papel,” Sci. For. Sci., vol. 40, no. 103, pp. 345-351, Jan. 2014.

[4] M. Shishehbor, M. R. Pouranian, and R. Imaninasab, "Evaluating the adhesion properties of crude oil fractions on mineral aggregates at different temperatures through reactive molecular dynamics," Pet. Sci. Technol., vol. 36, no. $24,2018$.

[5] M. Shishehbor, M. R. Pouranian, and M. G. Ramezani, "Molecular investigations on the interactions of Graphene, Crude oil fractions and mineral aggregates at low, medium and high temperatures," Pet. Sci. Technol.

[6] S. Merum, J. B. Veluru, and R. Seeram, "Functionalized carbon nanotubes in bio-world: Applications, limitations and future directions,” Mater. Sci. Eng. B Solid-State Mater. Adv. Technol., vol. 223, pp. 43-63, 2017.

[7] E. Lam, K. B. Male, J. H. Chong, A. C. W. Leung, and J. H. T. Luong, "Applications of functionalized and nanoparticlemodified nanocrystalline cellulose," Trends in Biotechnology, vol. 30, no. 5. pp. 283-290, 2012.

[8] J. Chen, L. Yan, W. Song, and D. Xu, "Interfacial characteristics of carbon nanotube-polymer composites: A review," Compos. Part A Appl. Sci. Manuf., vol. 114, pp. 149-169, 2018.

[9] I. H. Chen, W. Yang, and M. A. Meyers, "Leatherback sea turtle shell: a tough and flexible biological design," Acta Biomater., vol. 28, pp. 2-12, 2015.

[10] C.-Y. Sun and P.-Y. Chen, "Structural design and mechanical behavior of alligator (Alligator mississippiensis) osteoderms," Acta Biomater., vol. 9, no. 11, pp. 9049-9064, 2013.

[11] M. S. Hosseini, F. A. Cordisco, and P. D. Zavattieri, "Analysis of bioinspired non-interlocking geometrically patterned interfaces under predominant mode I loading,” J. Mech. Behav. Biomed. Mater., vol. 96, pp. 244-260, 2019.

[12] A. B. Reising, R. J. Moon, and J. P. Youngblood, "Effect of Particle Alignment on Mechanical Property of Neat Cellulose Nanocrystal Films," J. Sci. Technol. For. Prod. Process., vol. 2, no. 6, pp. 3-5, 2012.

[13] M. Shishehbor, F. L. Dri, R. J. Moon, and P. D. Zavattieri, “A continuum-based structural modeling approach for cellulose nanocrystals (CNCs)," J. Mech. Phys. Solids, vol. 111, pp. 308-332, 2018.

[14] M. Eftekhari, S. H. Ardakani, and S. Mohammadi, "An XFEM multiscale approach for fracture analysis of carbon nanotube reinforced concrete," Theor. Appl. Fract. Mech., vol. 72, pp. 64-75, 2014.

[15] M. J. Buehler, "Mesoscale modelling of mechanics of carbon nanotubes: Self-assembly, self-folding, and fracture," J. Mater. Res., vol. 21, no. 11, pp. 2855-2869, 2006.

[16] M. G. Ramezani and B. Golchinfar, "Mechanical Properties of Cellulose Nanocrystal (CNC) Bundles: Coarse-Grained Molecular Dynamic Simulation,” J. Compos. Sci., vol. 3, no. 2, p. 57, 2019.

[17] M. Shishehbor and P. D. Zavattieri, "Effects of interface properties on the mechanical properties of bio-inspired cellulose nanocrystal (CNC)-based materials," J. Mech. Phys. Solids, vol. 124, pp. 871-896, 2019.

[18] J. Leonardo, M. Shishehbor, and P. D. Zavattieri, “Mechanics of Crystalline Nano Cellulose Nanofilm,” 2017.

[19] R. Wagner, R. J. Moon, and A. Raman, "Mechanical properties of cellulose nanomaterials studied by contact resonance atomic force microscopy," Cellulose, vol. 23, no. 2, pp. 1031-1041, Apr. 2016.

[20] H. S. Esmaeeli, M. Shishehbor, W. J. Weiss, and P. D. Zavattieri, "A two-step multiscale model to predict early age strength development of cementitious composites considering competing fracture mechanisms," Constr. Build. Mater., vol. 208, pp. 577-600, 2019. 


\section{AUTHORS PROFILE}

Jim Huang was formerly an undergraduate student in mechanical Engineering at Portsmouth, United Kingdom. His research interest is on polymer, composite materials, molecular modeling and finite element modeling. He is currently pursuing an advanced degree in bioengineering.

Mehrdad Bor was formerly an undergraduate student in mechanical Engineering at Portsmouth, United Kingdom. His research interest is on composite materials, solid mechanics, continuum modeling, multiscale modeling and finite element modeling. He is currently a composite structural engineer. 